\title{
Eficácia, tolerabilidade e segurança do uso do sirolimo após o transplante renal
}

\author{
Sirolimus efficacy, tolerability, and safety for treatment after \\ kidney transplantation
}

\begin{abstract}
Autores
Nagilla lone Oliveira

Kelly Miyuki Harada

Gláucio Amaral

Spinelli

Sung In Park

Edison Luiz Mandia

Sampaio

Cláudia Rosso Felipe

Hélio Tedesco Silva

Júnior

José Osmar Medina

Pestana

Hospital do Rim e

Hipertensão - Fundação

Oswaldo Ramos -

Disciplina de Nefrologia

- UNIFESP - São Paulo,

$\mathrm{SP}$, Brasil
\end{abstract}

Data de submissão: 08/06/2009 Data de aprovação: 28/09/2009

Correspondência para: Hélio Tedesco Silva Junior Hospital do Rim e Hipertensão Rua Borges Lagoa, 960/11응 andar

São Paulo - SP - Brasil

CEP: 04038-002

E-mail: heliotedesco@hrim. com.br

Agência de fomento: Coordenação de Aperfeiçoamento de Pessoal de Nível Superior (Capes).

\section{Resumo}

Introdução: Sirolimo (SRL) é um imunossupressor com conhecida eficácia e perfil de segurança na profilaxia da rejeição aguda após o transplante renal. Objetivos: Avaliar eficácia, tolerabilidade e segurança do uso do SRL e de prednisona em associação a ciclosporina (CSA) ou tacrolimo (TAC) após o transplante renal. Metodologia: Estudo retrospectivo de 332 receptores de transplantes renais realizados entre 1999 e 2006. O desfecho primário foi a falha de tratamento, definida como a incidência cumulativa de rejeição aguda confirmada por biópsia (RACB), perda do enxerto, óbito ou descontinuação do SRL. Resultados: Dos 332 transplantes, $92 \%$ foram com doador vivo. A média de idade dos receptores foi de 37 anos, sendo $65 \%$ homens, $46 \%$ brancos e $6 \%$ diabéticos. SRL foi associado a CSA ou TAC em $70,8 \%$ e $29,2 \%$ dos pacientes. A incidência de falha de tratamento foi de $22,2 \%$ e de $47,8 \%$ no final do primeiro e do quinto ano de transplante, sem diferença entre pacientes recebendo CSA ou TAC. Ao final do quinto ano, as sobrevidas do paciente, do enxerto, do enxerto censorando o óbito e livre de RACB foram de $92,8 \%$, $86,1 \%, 92,7 \%$ e $82,2 \%$, respectivamente. O tratamento com SRL foi interrompido em $27,1 \%$ dos pacientes: $22,9 \%$ em razão de reações adversas e 3,3\% devido à ineficácia. Os principais motivos de suspensão do SRL foram dislipidemia $(6,0 \%)$, disfunção do enxerto $(5,2 \%)$, proteinúria $(4,5 \%)$, infecções $(1,5 \%)$, dificuldade de cicatrização $(1,2 \%)$ e anemia $(0,9 \%)$. Conclusão: $\mathrm{Na}$ população estudada, a eficácia e a segurança do SRL foram semelhantes quando combinado com CSA ou TAC. A tolerabilidade oral foi adequada considerando-se a relativa baixa taxa de interrupção do uso de SRL.

Palavras-chave: sirolimo, tolerância medicamentosa, eficácia, segurança, transplante de rim.

[J Bras Nefrol 2009;31(4):258-268]CElsevier Editora Ltda.

\section{Abstract}

Introduction: Sirolimus (SRL) is an immunosuppressive drug with confirmed efficacy and safety profile in the prophylaxis of acute rejection after renal transplantation. Objectives: To assess the efficacy, safety, and tolerability of SRL and prednisone in combination with cyclosporine (CSA) or tacrolimus (TAC) after renal transplantation. Methods: Retrospective study of 332 renal transplant recipients from 1999 to 2006. Primary outcome included treatment failure, defined as the cumulative incidence of biopsy-proven acute rejection, graft loss, death, or SRL discontinuation. Results: Living donors were the primary source of kidneys (92\%). Regarding the recipients, mean age was 37 years, $65 \%$ were males, $46 \%$ were white, and the prevalence of diabetes was $6 \%$. Sirolimus was combined with CSA and TAC in $70.8 \%$ and $29.2 \%$ of patients, respectively. Treatment failure rates at the first and fifth year of transplantation were $22.2 \%$ and $47.8 \%$, respectively, without difference between the groups receiving CSA and TAC. At five years, the survival rates were as follows: patient's, 92.8\%; graft's, $86.1 \%$; deathcensored graft's, $92.7 \%$; and free from biopsy-proven acute rejection, $82.2 \%$. Treatment with SRL was discontinued in $27.1 \%$ of the patients, due to adverse effects $(22.9 \%)$ and inefficacy $(3.3 \%)$. The main reasons for SRL discontinuation were as follows: dyslipidemia $(6 \%)$; graft dysfunction $(5.2 \%)$; proteinuria $(4.5 \%)$; infection $(1.5 \%)$; delayed wound healing $(1.2 \%)$; and anemia $(0.9 \%)$. Conclusion: In this cohort of patients, SRL efficacy and safety were similar when combined with either CSA or TAC. Oral tolerability was adequate, considering the relatively low SRL discontinuation rate.

Keywords: sirolimus, drug tolerability, efficacy, safety, renal transplantation. 


\section{INTRODUÇÃO}

Sirolimo (SRL) é um fármaco imunossupressor aprovado para a profilaxia da rejeição aguda após o transplante renal. Após penetrar na célula, o SRL se liga a uma outra proteína (FKBP12 - FK binding protein), formando um complexo fármaco-proteína que inibe uma outra proteína citoplasmática (mTOR - mamalian target of rapamycin) envolvida em uma complexa via de sinalização intracelular que coordena processos de crescimento, metabolismo, proliferação celular, autofagia e angiogênese. A inibição da mTOR promove a redução da síntese de proteínas, bloqueando a proliferação e diferenciação após a ativação celular. ${ }^{1}$

O fármaco apresenta rápida absorção oral e é submetido a metabolismo intestinal e hepático por um sistema de cooperação que inclui a glicoproteína-P e as enzimas do citocromo CYP3A. Isso determina, assim como ocorre com outros fármacos, uma grande variabilidade inter e intraindividual nas concentrações sanguíneas observadas em pacientes recebendo doses fixas de SRL. ${ }^{2}$ Há sinergismo entre SRL e ciclosporina (CSA), o que pode exacerbar a lesão renal induzida pela CSA em decorrência do aumento da sua concentração tanto no sangue ${ }^{3}$ quanto no tecido renal. Este resultado se deve às interações competitivas mútuas de ambos os fármacos como substrato para a glicoproteína-P e para as isoenzimas do CYP3A4 na célula luminal do intestino ou nas fases de distribuição, metabolização e depuração do fármaco. ${ }^{4}$

Em comparação com a azatioprina, a combinação de 2 ou $5 \mathrm{mg}$ de SRL com doses plenas de CSA resultou em menor incidência de rejeição aguda, ${ }^{5}$ mas maior incidência de nefrotoxicidade e hiperlipidemia. ${ }^{6,7}$ Como consequência, estratégias para reduzir a nefrotoxicidade foram investigadas.

Em receptores de doador vivo, utilizamos doses fixas de $2 \mathrm{mg}$ de SRL com redução rápida das doses de CSA. Nesse estudo, a incidência de rejeição aguda $(11,4 \%$ versus $14,3 \%, \mathrm{p}>0,05)$ e a função renal (creatinina média 1,8 $\pm 0,6$ versus $1,6 \pm 0,6 \mathrm{mg} / \mathrm{dL}, \mathrm{p}=0,23)$ foi similar àquelas observadas nos pacientes que receberam azatioprina e doses-padrão de CSA ao final do primeiro ano de transplante. ${ }^{8}$

Outras estratégias efetivas incluem a redução significativa da concentração-alvo ou a suspensão da CSA 3 meses após o transplante renal. Em um estudo multicêntrico nacional, essas duas estratégias foram eficazes na preservação da função renal após 1 ano de transplante (ritmo de filtração glomerular calculado: 49,5 versus $51,6 \mathrm{~mL} / \mathrm{min}, \mathrm{p}=0,603)$, sem que houvesse incremento estatisticamente significativo na incidência de rejeição aguda após a suspensão da CSA $(14,3 \%$ versus $22,5 \%, \mathrm{p}=0,152){ }^{9}$
Sirolimo também foi testado posteriormente, em combinação com tacrolimo (TAC). ${ }^{10}$ Esses fármacos competem pela ligação com a mesma imunofilina citoplasmática (FKBP12). Entretanto, acredita-se que a concentração dessa imunofilina seja suficientemente elevada para ligar ambos os fármacos nas doses utilizadas clinicamente. ${ }^{11}$ Assim como o que ocorre entre SRL e CSA, a interação farmacocinética entre SRL e TAC também pode ser observada. Há aumento de $59 \%$ nas concentrações de TAC em pacientes recebendo SRL e um aumento de $65 \%$ nas concentrações de SRL em pacientes em uso de TAC. ${ }^{3,12} \mathrm{Em}$ outro estudo prospectivo realizado no nosso centro, a combinação de doses fixas de $2 \mathrm{mg}$ de SRL com doses padrão de TAC apresentou eficácia comparável à combinação de micofenolato mofetil (MMF) com TAC na prevenção da rejeição aguda confirmada por biópsia (14 versus $12 \%, \mathrm{p}=1,0$ ). Entretanto, pacientes em uso de SRL apresentaram maior valor de creatinina $(1,6 \pm 0,5$ versus $1,4 \pm 0,3 \mathrm{mg} / \mathrm{dL}, \mathrm{p}=0,007)$, maior proporção de pacientes com proteinúria $(52,0$ versus $10,7 \%, p=0,041)$, maior concentração média de proteína na amostra isolada de urina $(0,3 \pm 0,5$ versus $0,1 \pm 0,2 \mathrm{~g} / \mathrm{L}, \mathrm{p}=0,012)$, maior concentração média de colesterol $(217$ versus $190 \mathrm{mg} / \mathrm{dL}, \mathrm{p}=0,030)$ e maior proporção de pacientes prematuramente descontinuados da terapia inicial ao final do primeiro ano do transplante. Esses dados sugerem a necessidade de estudos utilizando doses reduzidas de TAC em combinação com SRL. ${ }^{13}$

O uso clínico de SRL também tem sido explorado em pacientes que apresentam disfunção crônica do enxerto, utilizando-se várias estratégias. ${ }^{14} \mathrm{~A}$ conversão de CSA para MMF em receptores de transplante renal recebendo SRL e com diagnóstico de disfunção crônica do enxerto determinou, após 3 meses da conversão, a redução dos valores de creatinina $(2,1 \pm$ $1,0$ versus $1,7 \pm 0,4 \mathrm{mg} / \mathrm{dL}, \mathrm{p}<0,001)$ e o aumento da depuração de creatinina $(51,9 \pm 19,2$ versus 59,5 $\pm 16,1 \mathrm{~mL} / \mathrm{min}, \mathrm{p}<0,01)$. Houve, também, redução significativa nas concentrações de colesterol $(280 \pm$ 68 versus $217 \pm 49, \mathrm{p}=0,009)$ e triglicérides $(400 \pm$ 228 versus $205 \pm 75, \mathrm{p}=0,013) .{ }^{15}$

Além da eficácia comprovada na prevenção da rejeição aguda em diversas associações já testadas, o uso do SRL está associado à menor incidência de infecção por citomegalovírus e de neoplasias após o transplante renal. ${ }^{16}$ Por outro lado, além da hiperlipidemia e da potencialização dos efeitos nefrotóxicos dos inibidores da calcineurina, o SRL pode apresentar efeito tóxico sobre as células epiteliais tubulares ${ }^{17}$ induzir glomeruloesclerose segmentar e 
focal ${ }^{18}$ e acarretar proteinúria. ${ }^{17} \mathrm{~A}$ inibição da fosforilação da lipina, proteína-alvo da insulina, justifica a interferência do SRL sobre o controle do metabolismo de carboidratos e lipídios, podendo aumentar a incidência de diabetes mellitus após o transplante. ${ }^{19}$ Os efeitos do SRL sobre a cicatrização, de forma geral, e sobre a formação de linfocele, em particular, são reconhecidos e estão associados a suas propriedades antiproliferativas e seus efeitos inibitórios na angiogênese e na fibrose, interferindo com os mecanismos necessários para a cicatrização da ferida operatória. ${ }^{20}$

A diversidade de indicações terapêuticas e de reações adversas dificulta a determinação da melhor forma de utilização clínica desse medicamento imunossupressor. ${ }^{21,22}$ Este estudo visa avaliar, retrospectivamente, regimes imunossupressores utilizando SRL, desenvolvidos de forma sistemática nos últimos 10 anos em nosso centro. Essa avaliação crítica é fundamental para o desenho e a condução de novos estudos clínicos visando à utilização racional desse agente imunossupressor após o transplante renal.

\section{Métodos}

\section{DESENHO do ESTUdo}

Este é um estudo retrospectivo realizado por meio da análise de dados obtidos de prontuários de receptores de transplante renal realizado com doador vivo ou falecido, entre 10/06/1999 e 20/09/2006. O estudo foi aprovado pelo comitê de ética em pesquisa da UNIFESP.

\section{POPULAÇÃO - CRITÉRIOS DE INCLUSÃO E EXCLUSÃO}

Foram incluídos todos os receptores do primeiro transplante renal que receberam, como parte do esquema imunossupressor inicial após o transplante, SRL associado a CSA ou TAC e prednisona (PRED). Os dados foram coletados até 20/12/2007 ou até a perda do enxerto, óbito ou perda de seguimento do paciente. Os pacientes que interromperam o uso de qualquer um dos medicamentos imunossupressores continuaram a fazer parte do estudo. Os pacientes que receberam qualquer forma de terapia de indução foram excluídos.

\section{AVALIAÇÕES E DEFINIÇÕES DO ESTUDO}

Dados demográficos foram obtidos de todos os pacientes antes do transplante renal. Avaliações anuais até o quinto ano após o transplante incluíram dose e concentração dos fármacos imunossupressores, dados bioquímicos, hematológicos e urinários. Foram identificados todos os motivos que levaram à interrupção definitiva do uso do SRL.
Rejeição aguda confirmada por biópsia (RACB) foi definida como toda disfunção aguda do enxerto cuja análise histológica da biópsia pôde ser classificada de acordo com os critérios de Banff, 1997. A perda do enxerto renal foi definida como a necessidade de retorno para diálise ou a realização de um novo transplante. Perda de seguimento foi caracterizada pela ausência de informações mínimas por mais de 11 meses seguidos.

\section{IMUNOSSUPRESSÃO}

Todos os pacientes receberam CSA ou TAC em combinação com SRL e PRED. Metilprednisolona (1 g) foi administrada a todos os pacientes durante o período intraoperatório. A dose inicial de PRED foi de $30 \mathrm{mg} / \mathrm{dia}$, sendo reduzida progressivamente até atingir $10 \mathrm{mg} / \mathrm{dia}$ no terceiro mês após o transplante. A dose de ataque de SRL foi de $15 \mathrm{mg}$ (CSA, $\mathrm{n}=70$; TAC, $\mathrm{n}=0$ ) ou de $6 \mathrm{mg}$ (CSA, $\mathrm{n}=165$; TAC, $\mathrm{n}=97$ ), administrada no primeiro dia após o transplante, seguida de doses diárias fixas de $5 \mathrm{mg}$ ou $2 \mathrm{mg}$ até o sétimo dia após o transplante. Posteriormente, a dose do fármaco foi ajustada para que fossem obtidas concentrações sanguíneas de SRL entre 5 e $20 \mathrm{ng} / \mathrm{mL}$. As concentrações de SRL foram determinadas em amostras de sangue total por cromatografia. ${ }^{22}$

A dose inicial de TAC foi de $0,1 \mathrm{a} 0,15 \mathrm{mg} / \mathrm{kg}$ e foi ajustada para que fosse obtida uma concentração sanguínea entre 10 e $15 \mathrm{ng} / \mathrm{mL}$ (até 30 dias), 8 e $12 \mathrm{ng} / \mathrm{mL}$ (30-90 dias) e 5 e $10 \mathrm{ng} / \mathrm{mL}$ (> 90 dias). O ensaio IMx Tacro II (Abbott, Abbott Park, IL, EUA) foi utilizado para medir as concentrações de TAC em amostras de sangue total.

A dose inicial de CSA foi de 8 a $10 \mathrm{mg} / \mathrm{kg}$ e foi ajustada para que fossem obtidas concentrações sanguíneas entre 150 e $200 \mathrm{ng} / \mathrm{mL}$ (até 30 dias), 100 e $150 \mathrm{ng} / \mathrm{mL}$ (30-90 dias) e 50 e $100 \mathrm{ng} / \mathrm{mL}$ (> 90 dias). As concentrações de CSA foram medidas em amostras de sangue total por meio do método imunofluorescência polarizada (AxSYM - Abbott Laboratories, IL).

O regime terapêutico pôde ser alterado, com a interrupção transitória ou definitiva de qualquer um dos medicamentos, inclusive SRL, por qualquer motivo, seja ele a falta de tolerabilidade oral, a falta de eficácia ou a presença de reação adversa.

\section{Desfechos}

PRIMÁRIO

O desfecho primário desse estudo foi a falha do tratamento determinada pela ocorrência cumulativa de rejeição aguda confirmada por biópsia, interrupção definitiva do uso de SRL, perda do enxerto, óbito do paciente ou perda de seguimento aferida entre o primeiro e o quinto anos após o transplante. 


\section{SECUNDÁRIOS}

Os desfechos secundários foram: (1) a proporção cumulativa de pacientes livres de rejeição aguda confirmada por biópsia; (2) a proporção cumulativa de pacientes livres de interrupção definitiva do uso de SRL; (3) a sobrevida cumulativa do paciente; (4) a sobrevida cumulativa do enxerto; e (5) a sobrevida cumulativa do enxerto censorando o óbito.

As causas de interrupção definitiva do uso do SRL foram identificadas e associadas à falta de eficácia ou à toxicidade. Avaliações bioquímicas de segurança incluíram a medida da função renal pelos indicadores creatinina $(\mathrm{mg} / \mathrm{dL})$, colesterol total $(\mathrm{mg} / \mathrm{dL})$, triglicérides $(\mathrm{mg} / \mathrm{dL})$, glicemia $(\mathrm{mg} / \mathrm{dL})$ e concentração de proteína em amostra isolada de urina $(\mathrm{g} / \mathrm{L})$ determinada por método colorimétrico (Vermelho de Pirogalol). A avaliação hematológica incluiu a concentração de hemoglobina $(\mathrm{g} / \mathrm{dL})$ e a contagem de glóbulos brancos $\left(\mathrm{N} / \mathrm{mm}^{3}\right)$ e plaquetas $\left(\mathrm{N} / \mathrm{mm}^{3}\right)$.

\section{ANÁLISE ESTATÍSTICA}

As variáveis contínuas foram apresentadas como média e desvio-padrão e as diferenças entre os grupos foram identificadas utilizando-se o teste $t$ de Student não pareado entre dois grupos e análise de variância (ANOVA) para mais de dois grupos. As variáveis categóricas foram apresentadas como frequência e porcentagem, e as diferenças foram identificadas utilizando-se o teste qui-quadrado.

A análise dos desfechos primário ou secundário foi realizada por intenção de tratamento com base no regime imunossupressor inicial (CSA ou TAC) e incluíram todos os pacientes submetidos a transplante renal e que receberam pelo menos uma dose de SRL. As informações de pacientes preenchendo o critério de perda de seguimento foram censoradas por seu último dia de registro de seguimento ambulatorial.

As curvas de sobrevida foram obtidas pelo produto de Kaplan-Meier, com teste Log Rank para avaliar diferenças estatísticas entre os grupos de tratamento. A análise estatística foi realizada com o programa SPSS v. 7.5 (SPSS Inc., Chicago, IL, EUA). A diferença estatística foi definida com valor $\mathrm{p}<0,05$.

\section{Resultados}

\section{DEMOGRAFIA}

Foram incluídos neste estudo 332 receptores de transplante renal. A população estudada foi jovem, predominantemente masculina $(65 \%)$ e de etnia branca $(46 \%)$. Os receptores de etnia negra compreenderam $20 \%$ do total e diabetes mellitus foi a etiologia da doença renal crônica em apenas 6\% dos pacientes estudados. O tratamento dialítico mais prevalente foi a hemodiálise e o tempo médio em diálise foi de 25 meses. O transplante renal foi realizado majoritariamente com doador vivo $(92 \%)$. Houve predominância do uso de CSA $(70,8 \%)$ em comparação ao uso de TAC $(29,2 \%)$, apesar de não terem sido identificadas diferenças estatisticamente significativas comparando-se as duas populações. As características demográficas da população estudada estão apresentadas na Tabela 1.

\section{IMUNOSSUPRESSÃO}

Ao final do primeiro ano de transplante, a média da concentração de CSA foi de $78 \mathrm{ng} / \mathrm{mL}$, e foi obtida utilizando-se uma dose média de CSA de 132 $\mathrm{mg} /$ dia. Da mesma forma, a média da concentração de TAC foi de $6,2 \mathrm{ng} / \mathrm{mL}$, e foi obtida utilizando-se uma dose média de TAC de $6,4 \mathrm{mg} / \mathrm{dia}$. A dose $(3,5$ versus $3,9 \mathrm{mg} / \mathrm{dia})$ e a concentração média $(11,4$ versus $10,1 \mathrm{ng} / \mathrm{mL}$ ) de SRL foram semelhantes entre os pacientes que receberam CSA ou TAC, respectivamente (Tabela 2). As doses de PRED também foram semelhantes entre os grupos. O uso de PRED foi eletivamente interrompido em $24(24,7 \%)$ dos pacientes que receberam TAC e SRL. Não foram observadas mudanças significativas nas doses ou concentrações dos fármacos entre o primeiro e o quinto anos após o transplante.

A avaliação de adesão ao regime imunossupressor inicial está demonstrada na Tabela 3. Dos 235 pacientes que receberam inicialmente a combinação de SRL e CSA, 46 tiveram o tratamento interrompido antes do final do primeiro ano de transplante, seja eletivamente ou pela ocorrência de perda do enxerto, óbito ou perda de seguimento. Dos 189 pacientes que completaram o primeiro ano com a possibilidade de ainda estar recebendo a combinação inicial, em 35 pacientes uma das drogas foi descontinuada, resultando em uma taxa de adesão ao tratamento de $81,5 \%$ ao final do primeiro ano de transplante. Nos anos seguintes, as taxas de adesão ao tratamento variaram entre $53 \%$ e $89 \%$. Da mesma forma, dos 97 pacientes que receberam inicialmente a combinação de SRL e TAC, 74 completaram o primeiro ano com a possibilidade de ainda estar recebendo essa combinação. Em 19 desses pacientes o uso de uma das drogas foi interrompido, resultando em uma taxa de adesão ao tratamento de $68,9 \%$ ao final do primeiro ano de transplante. As taxas de adesão ao tratamento variaram entre $87 \%$ e $95 \%$ nos anos seguintes (Tabela 3). 


\begin{tabular}{|c|c|c|c|}
\hline \multirow[t]{2}{*}{ Características } & \multicolumn{3}{|c|}{ CARACTERÍSTICAS DEMOGRÁFICAS DE 332 TRANSPLANTADOS RENAIS } \\
\hline & $\begin{array}{c}\text { TOTAL } \\
(\mathrm{n}=332)\end{array}$ & $\begin{array}{c}\text { CSA } \\
(n=235)\end{array}$ & $\begin{array}{c}\text { TAC } \\
(n=97)\end{array}$ \\
\hline Idade (anos) & $37 \pm 11$ & $38 \pm 11$ & $36 \pm 11$ \\
\hline \multicolumn{4}{|l|}{ Sexo, n (\%) } \\
\hline Masculino & $217(65)$ & $158(67)$ & $59(61)$ \\
\hline Feminino & $115(35)$ & $77(33)$ & 38 (39) \\
\hline \multicolumn{4}{|l|}{ Etnia, $n(\%)$} \\
\hline Branco & $152(46)$ & $103(44)$ & $49(51)$ \\
\hline Negro & $66(20)$ & $50(21)$ & $16(16)$ \\
\hline Mulato & $104(31)$ & 77 (33) & $27(28)$ \\
\hline Asiático & $10(3)$ & $5(2)$ & $5(5)$ \\
\hline \multicolumn{4}{|l|}{ Causa da DRC, $\mathrm{n}(\%)$} \\
\hline Gromeluronefrite crônica & $57(17)$ & $46(19)$ & $11(11)$ \\
\hline Hipertensão & $60(18)$ & $51(22)$ & $12(12)$ \\
\hline Diabetes mellitus & $21(6)$ & $16(7)$ & $5(5)$ \\
\hline Indeterminada & $129(39)$ & $67(28)$ & $62(63)$ \\
\hline Outros & $65(20)$ & $56(24)$ & $9(9)$ \\
\hline \multicolumn{4}{|l|}{ Tratamento prévio, n (\%) } \\
\hline Hemodiálise & 306 (92) & $219(93)$ & $87(90)$ \\
\hline Diálise peritoneal & $18(6)$ & $12(5)$ & $6(6)$ \\
\hline Conservador & $8(2)$ & $4(2)$ & $4(4)$ \\
\hline Tempo de diálise (meses) & $25,0 \pm 21,6$ & $24,3 \pm 20,2$ & $28 \pm 31,6$ \\
\hline Idade do doador (anos) & $42 \pm 11$ & $42 \pm 11$ & $43 \pm 10$ \\
\hline \multicolumn{4}{|l|}{ Tipo de doador, $\mathrm{n}(\%)$} \\
\hline Vivo & 306 (92) & $221(94)$ & $85(88)$ \\
\hline Falecido & $26(8)$ & $14(6)$ & $12(12)$ \\
\hline
\end{tabular}

DRC: doença renal crônica; p > 0,05 CSA (ciclosporina) versus TAC (tacrolimo).

Tabela 2 DOSES E CONCENTRAÇÕES DOS IMUNOSSUPRESSORES NO FINAL DO PRIMEIRO ANO DE TRANSPLANTE RENAL

\begin{tabular}{|c|c|c|c|c|c|}
\hline & \multicolumn{2}{|c|}{ ICN } & \multicolumn{2}{|c|}{ SRL } & \multirow{2}{*}{$\begin{array}{c}\text { PRED } \\
\text { Dose } \\
\text { (mg/dia) }\end{array}$} \\
\hline & $\begin{array}{l}\text { Dose } \\
\text { (mg/dia) }\end{array}$ & $\begin{array}{c}\text { Concentração } \\
(\mathrm{ng} / \mathrm{mL})\end{array}$ & $\begin{array}{c}\text { Dose } \\
\text { (mg/dia) }\end{array}$ & $\begin{array}{c}\text { Concentração } \\
(\mathrm{ng} / \mathrm{mL})\end{array}$ & \\
\hline $\operatorname{CSA}(n=189)$ & $132,4 \pm 72,1$ & $77,9 \pm 56,4$ & $3,5 \pm 2,3$ & $11,4 \pm 5,7$ & $8,8 \pm 4,9$ \\
\hline TAC $(n=74)$ & $6,4 \pm 3,7$ & $6,2 \pm 2,6$ & $3,9 \pm 2,6$ & $10,1 \pm 5,1$ & $9,1 \pm 5,1$ \\
\hline
\end{tabular}

ICN: inibidor da calcineurina; SRL: sirolimo; PRED: prednisona; CSA: ciclosporina; TAC: tacrolimo.

\section{DESFECHO COMPOSTO PRIMÁRIO}

A incidência de falha do tratamento, definida como a ocorrência de rejeição aguda comprovada por biópsia, perda do enxerto, óbito ou descontinuação do sirolimo, foi de $22,2 \%$ ao final do primeiro ano, e de $47,8 \%$ ao final do quinto ano do transplante, sem diferenças significativas entre os pacientes que receberam CSA ou TAC (Tabela 4, Figura 1).

\section{DESFECHOS SECUNDÁRIOS}

As incidências cumulativas dos desfechos secundários individuais ou combinados estão demonstradas na Tabela 4. A sobrevida livre de rejeição aguda confirmada por biópsia foi de $87,9 \%$ e de $82,2 \%$ no final do primeiro e do quinto anos após o transplante, sendo semelhante entre os pacientes que receberam CSA ou TAC. A sobrevida livre da interrupção do uso do 
Tabela 3 NÚMERO E PROPORÇÃO DE PACIENTES QUE CONTINUARAM COM A TERAPIA POR INTENÇÃO DE TRATAMENTO AO LONGO DE 5 ANOS DE TRANSPLANTE RENAL

\begin{tabular}{|c|c|c|c|c|c|}
\hline Ano após o transplante & $\begin{array}{c}1 \\
n(\%)\end{array}$ & $\begin{array}{c}2 \\
n(\%)\end{array}$ & $\begin{array}{c}3 \\
n(\%)\end{array}$ & $\begin{array}{c}4 \\
n(\%)\end{array}$ & $\begin{array}{c}5 \\
n(\%)\end{array}$ \\
\hline CSA/SRL & 235 & 154 & 102 & 59 & 30 \\
\hline Interrupção do tratamento & $46(19,6)$ & $8(5,2)$ & $2(2,0)$ & $2(3,4)$ & $3(10,0)$ \\
\hline Eletiva (CSA) & $33(14,0)$ & 0 & 0 & 0 & 0 \\
\hline Perda & $6(2,6)$ & $4(2,6)$ & $2(2,0)$ & 0 & $2(6,7)$ \\
\hline Óbito & $5(2,1)$ & $4(2,6)$ & 0 & 0 & 0 \\
\hline Perda de seguimento & $2(0,9)$ & 0 & 0 & $2(3,4)$ & $1(3,3)$ \\
\hline Total em intenção de tratamento & $189(79,4)$ & $146(94,8)$ & $100(98,0)$ & $57(96,6)$ & $27(90,0)$ \\
\hline Descontinuação & $35(18,5)$ & $44(30,1)$ & $41(41,0)$ & $27(47,4)$ & $3(11,1)$ \\
\hline CSA & $16(8,5)$ & $26(17,8)$ & $33(33,0)$ & $22(38,6)$ & $2(7,4)$ \\
\hline SRL & $19(10,0)$ & $18(12,3)$ & $8(8,0)$ & $5(8,8)$ & $1(3,7)$ \\
\hline Total em tratamento & $154(81,5)$ & $102(69,9)$ & $59(59,0)$ & $30(52,6)$ & $24(88,9)$ \\
\hline TAC/SRL & 97 & 55 & 46 & 42 & - \\
\hline Interrupção do tratamento & $23(23,7)$ & $2(3,6)$ & $2(4,4)$ & 0 & - \\
\hline Eletiva (TAC) & $20(20,6)$ & 0 & 0 & 0 & - \\
\hline Perda & 0 & 0 & 0 & 0 & - \\
\hline Óbito & $2(2,1)$ & $1(1,8)$ & $1(2,2)$ & 0 & - \\
\hline Perda de seguimento & $1(1,0)$ & $1(1,8)$ & $1(2,2)$ & 0 & - \\
\hline Total em intenção de tratamento & $74(76,3)$ & $53(96,4)$ & $44(95,6)$ & $42(100)$ & \\
\hline Descontinuação & $19(25,7)$ & $7(13,2)$ & $2(4,5)$ & $3(7,2)$ & - \\
\hline TAC & $5(6,8)$ & $2(3,8)$ & 0 & $1(2,4)$ & - \\
\hline SRL & $14(18,9)$ & $5(9,4)$ & $2(4,5)$ & $2(4,8)$ & - \\
\hline Total em tratamento & $55(74,3)$ & $46(86,8)$ & $42(95,4)$ & $39(92,8)$ & - \\
\hline
\end{tabular}

SRL: sirolimo; PRED: prednisona; CSA: ciclosporina; TAC: tacrolimo.

Figura 1. Curvas de sobrevida cumulativas, global e por tipo de inibidor de calcineurina, livres de rejeição aguda comprovada por biópsia, perda do enxerto, óbito ou descontinuação do tratamento com sirolimo; p > 0,05 CSA (ciclosporina) versus TAC (tacrolimo).

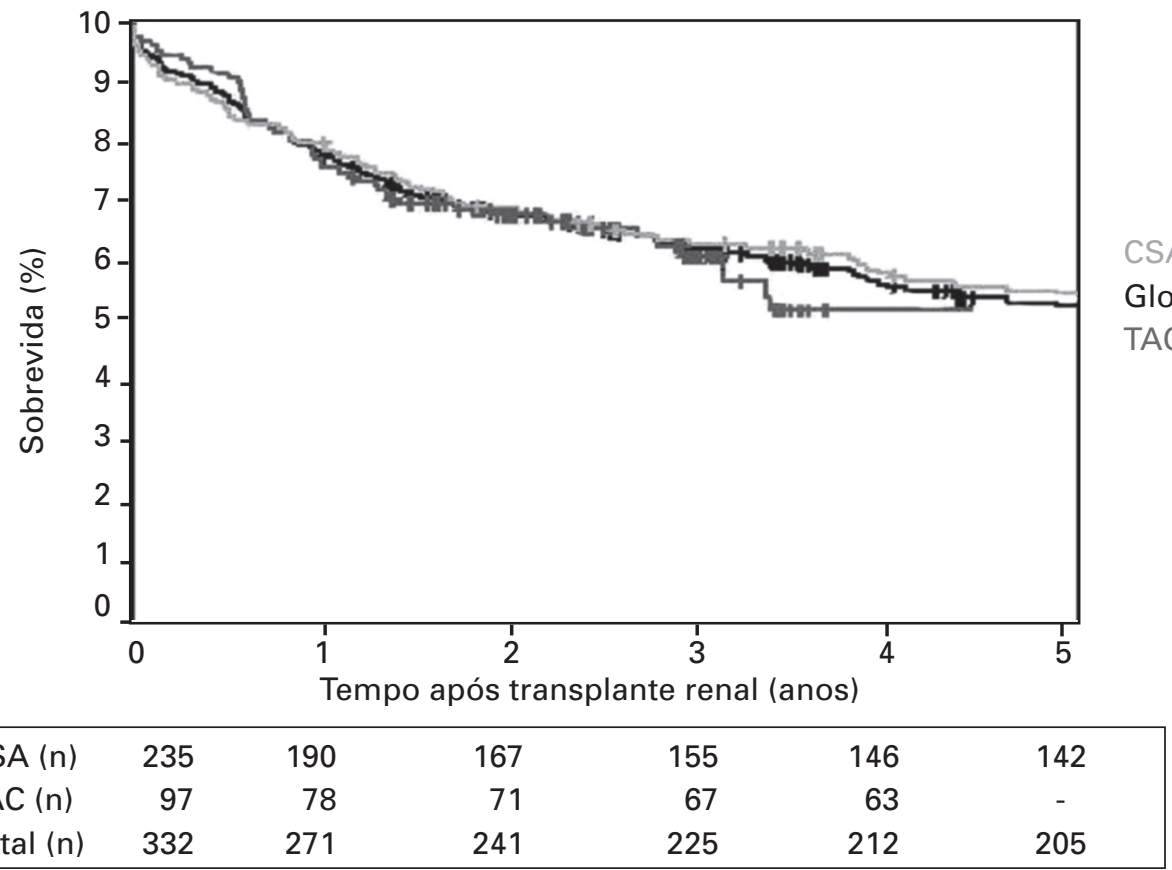


SRL foi de $88 \%$ no final do primeiro ano $(89,5 \%$ no grupo CSA versus $84,5 \%$ do grupo TAC, $\mathrm{p}>0,05)$ e de $66,8 \%$ no quinto ano após o transplante $(70,8 \%$ no grupo CSA versus $62,0 \%$ do grupo TAC, $\mathrm{p}>0,05)$. Até o final do quinto ano de transplante, $92,8 \%$ dos pacientes estavam vivos, sendo que $86,1 \%$ apresentavam enxerto funcionante e $73,8 \%$ não haviam apresentado rejeição aguda confirmada por biópsia (Tabela 4).

A interrupção do tratamento com SRL ocorreu em $27,1 \%$ dos pacientes. Em $22,9 \%$ dos pacientes, a presença de reações adversas determinou a interrupção do tratamento com SRL. Entre as causas principais se destacaram a dislipidemia $(6,0 \%)$, a disfunção do enxerto $(5,2 \%)$ e a proteinúria $(4,5 \%)$. A falta de eficácia foi responsável pela interrupção do uso do SRL em 3,3\% dos pacientes. Não foi observada diferença nas proporções de pacientes que usavam CSA ou TAC cujo tratamento com SRL foi interrompido, seja por toxicidade ou falta de eficácia (Tabela 5).

\section{Tabela 4 ANÁLISE DA EFICÁCIA DE REGIMES IMUNOSSUPRESSORES UTILIZANDO SIROLIMO APÓS TRANSPLANTE RENAL}

Sobrevida \%
Tempo após o transplante renal (anos)

$2 \quad 3 \quad 4 \quad 5$

$(\mathrm{n}=332) \quad(\mathrm{n}=323) \quad(\mathrm{n}=291) \quad(\mathrm{n}=251) \quad(\mathrm{n}=218)$

Livre de RACB, perda, óbito ou descontinuação RACB

77,8

10,5

68,2

$=291)$

Perda de enxerto

0,9

Óbito

Descontinuação do sirolimo

1,5

Censorados

Livre de RACB, perda ou óbito

Livre de RACB ou perda

Livre de RACB

Livre de descontinuação

Livre de perda ou óbito (enxerto)

Livre de óbito (paciente)

Livre de perda (enxerto com óbito censorado)

RACB: rejeição aguda comprovada por biópsia; $p>0,05$ ciclosporina versus tacrolimo.

\begin{tabular}{|cccc|}
\hline 68,2 & 62,2 & 55,7 & 52,2 \\
\hline 1,5 & 1,0 & 1,2 & 0,4 \\
\hline 0,6 & 0,3 & 0 & 0 \\
\hline 1,2 & 0,7 & 0,4 & 0 \\
\hline 6,2 & 3,4 & 3,6 & 2,3 \\
\hline 8,0 & 12,7 & 12,7 & 4,1 \\
\hline 81,2 & 78,7 & 75,8 & 73,8 \\
\hline 84,2 & 82,7 & 81,0 & 79,9 \\
\hline 86,4 & 85,2 & 83,6 & 82,2 \\
\hline 80,9 & 76,1 & 70,0 & 66,8 \\
\hline 92,0 & 89,7 & 88,1 & 86,1 \\
\hline 95,6 & 94,8 & 93,5 & 92,8 \\
\hline 96,2 & 94,7 & 94,2 & 92,7 \\
\hline
\end{tabular}

\section{Tabela 5 CAUSAS DE DESCONTINUAÇÃO DO TRATAMENTO COM SIROLIMO DURANTE 5 ANOS DE} TRANSPLANTE RENAL

\begin{tabular}{|c|c|c|c|c|}
\hline & & $\begin{array}{c}\text { Total n (\%) } \\
n=332\end{array}$ & $\begin{array}{c}\text { CSA n (\%) } \\
n=235\end{array}$ & $\begin{array}{c}\text { TAC n (\%) } \\
n=97\end{array}$ \\
\hline Ineficácia & & $11(3,3)$ & $10(4,3)$ & $1(1,0)$ \\
\hline Toxicidade & & $76(22,9)$ & $51(21,7)$ & $25(25,8)$ \\
\hline & Dislipidemia & $20(6,0)$ & $15(6,4)$ & $5(5,2)$ \\
\hline & Disfunção do enxerto & $17(5,2)$ & $9(3,9)$ & $8(8,3)$ \\
\hline & Proteinúria & $15(4,5)$ & $12(5,1)$ & $3(3,1)$ \\
\hline & Infecção & $5(1,5)$ & $4(1,7)$ & $1(1,0)$ \\
\hline & Dificuldade de cicatrização & $4(1,2)$ & $1(0,4)$ & $3(3,1)$ \\
\hline & Anemia & $3(0,9)$ & $3(1,3)$ & 0 \\
\hline & Neoplasia & $1(0,3)$ & $1(0,4)$ & 0 \\
\hline & Aumento de creatina quinase & $1(0,3)$ & $1(0,4)$ & 0 \\
\hline & Colite crônica inespecífica & $1(0,3)$ & 0 & $1(1,0)$ \\
\hline & Intolerância gastrointestinal & $1(0,3)$ & $1(0,4)$ & 0 \\
\hline & Pneumopatia (toxicidade pulmonar) & $1(0,3)$ & $1(0,4)$ & 0 \\
\hline & Síndrome hemolítica urêmica & $1(0,3)$ & 0 & $1(1,0)$ \\
\hline & Neuropatia periférica & $1(0,3)$ & 0 & $1(1,0)$ \\
\hline & Gestação do paciente ou cônjuge & $5(1,5)$ & $3(1,3)$ & $2(2,1)$ \\
\hline Outros & & $3(0,9)$ & $2(0,8)$ & $1(1,0)$ \\
\hline TOTAL & & $90(27,1)$ & $63(26,8)$ & $27(27,8)$ \\
\hline
\end{tabular}

$p>0,05$ CSA (ciclosporina) versus TAC (tacrolimo). 


\section{AvaliaÇÃo BIOQuímicA e hematológica}

A função renal do enxerto permaneceu estável entre o primeiro e o quinto anos de transplante. A partir do terceiro ano, a média da creatinina dos pacientes recebendo TAC foi maior em comparação com a dos pacientes recebendo CSA (Tabela 6). A concentração média de proteína urinária foi de $0,52 \pm 1,0 \mathrm{~g} / \mathrm{L}$ no final do primeiro ano, aumentando para $0,82 \pm 1,1 \mathrm{~g} / \mathrm{L}$ no quinto ano após o transplante. Não houve diferença na concentração de proteína urinária comparando-se os pacientes recebendo CSA com os que recebiam TAC.

No final do primeiro ano, as concentrações médias de colesterol e triglicérides foram de 239 e $277 \mathrm{mg} / \mathrm{dL}$, respectivamente, e não se elevaram posteriormente. Enquanto as concentrações médias de colesterol foram maiores até o terceiro ano do transplante nos pacientes recebendo CSA, essa diferença foi observada somente ao final do primeiro ano, pela análise das concentrações médias de triglicérides. A concentração de glicose foi $101 \pm 52 \mathrm{mg} / \mathrm{dL}$ no final do primeiro ano, permaneceu estável até o final do seguimento e não foi observada diferença entre os pacientes recebendo CSA ou TAC (Tabela 6).

As concentrações médias de hemoglobina foram de $13,9 \pm 2,5 \mathrm{~g} / \mathrm{dL}$ no final do primeiro ano e ficaram estáveis até o quinto ano $(13,2 \pm 2,1 \mathrm{~g} / \mathrm{dL})$. A proporção de pacientes com concentração de hemoglobina inferior a $10 \mathrm{~g} / \mathrm{dL}$ não foi diferente comparando-se pacientes recebendo CSA com aqueles que recebiam TAC (4 versus $7 \%, \mathrm{p}>0,05)$, respectivamente. Não

Tabela 6 AVALIAÇÃo BIOQUÍMICA E HEMATOLÓGICA APÓS O TRANSPLANTE RENAL

\begin{tabular}{|c|c|c|c|c|c|}
\hline Ano após o transplante & 1 & 2 & 3 & 4 & 5 \\
\hline \multicolumn{6}{|l|}{ Creatinina $(\mathrm{mg} / \mathrm{dL})$} \\
\hline TOTAL & $1,69 \pm 0,9$ & $1,71 \pm 0,7$ & $1,76 \pm 0,8$ & $1,71 \pm 0,7$ & $1,73 \pm 0,6$ \\
\hline CSA & $1,75 \pm 1,1$ & $1,72 \pm 0,7$ & $1,69 \pm 0,6$ & $1,67 \pm 0,5$ & $1,73 \pm 0,6$ \\
\hline TAC $^{b}$ & $1,53 \pm 0,5$ & $1,70 \pm 0,7$ & $2,15 \pm 1,2^{\mathrm{a}}$ & $2,12 \pm 1,3^{a}$ & - \\
\hline \multicolumn{6}{|l|}{ Proteína urinária (g/L) } \\
\hline TOTAL $^{\mathrm{b}}$ & $0,52 \pm 1,0$ & $0,42 \pm 0,73$ & $0,56 \pm 0,82$ & $0,7 \pm 0,9$ & $0,82 \pm 1,1$ \\
\hline $\mathrm{CSA}^{\mathrm{b}}$ & $0,51 \pm 1,1$ & $0,40 \pm 0,7$ & $0,54 \pm 0,8$ & $0,69 \pm 0,9$ & $0,82 \pm 1,1$ \\
\hline TAC $^{b}$ & $0,54 \pm 1,1$ & $0,47 \pm 0,8$ & $0,67 \pm 0,8$ & $0,75 \pm 0,8$ & - \\
\hline \multicolumn{6}{|l|}{ Colesterol (mg/dL) } \\
\hline TOTAL & $239 \pm 71$ & $238 \pm 58$ & $239 \pm 65$ & $235 \pm 62$ & $225 \pm 56$ \\
\hline $\mathrm{CSA}^{\mathrm{b}}$ & $249 \pm 71$ & $245 \pm 59$ & $245 \pm 65$ & $238 \pm 63$ & $225 \pm 56$ \\
\hline TAC & $216 \pm 64^{a}$ & $205 \pm 44^{a}$ & $204 \pm 54^{a}$ & $207 \pm 49$ & - \\
\hline \multicolumn{6}{|l|}{ Triglicérides (mg/dL) } \\
\hline TOTAL & $277 \pm 186$ & $275 \pm 190$ & $281 \pm 190$ & $240 \pm 164$ & $251 \pm 249$ \\
\hline CSA & $293 \pm 192$ & $286 \pm 199$ & $293 \pm 193$ & $232 \pm 161$ & $251 \pm 249$ \\
\hline TAC & $239 \pm 164^{a}$ & $226 \pm 135$ & $206 \pm 158$ & $320 \pm 184$ & - \\
\hline \multicolumn{6}{|l|}{ Glicose (mg/dL) } \\
\hline TOTAL & $101 \pm 52$ & $102 \pm 46$ & $104 \pm 54$ & $104 \pm 45$ & $103 \pm 41$ \\
\hline CSA & $102 \pm 57$ & $103 \pm 49$ & $100 \pm 37$ & $101 \pm 40$ & $103 \pm 41$ \\
\hline TAC & $99 \pm 40$ & $98 \pm 37$ & $120 \pm 101$ & $119 \pm 69$ & - \\
\hline \multicolumn{6}{|l|}{ Hemoglobina (g/dL) } \\
\hline TOTAL ${ }^{\mathrm{b}}$ & $13,9 \pm 2,5$ & $13,6 \pm 2,1$ & $13,4 \pm 2,1$ & $13,1 \pm 1,9$ & $13,2 \pm 2,1$ \\
\hline CSA & $13,9 \pm 2,5$ & $13,6 \pm 2,2$ & $13,5 \pm 2,1$ & $13,0 \pm 1,9$ & $13,2 \pm 2,1$ \\
\hline TAC & $14,0 \pm 2,4$ & $13,7 \pm 1,9$ & $12,8 \pm 2,0$ & $13,7 \pm 2,0$ & - \\
\hline \multicolumn{6}{|c|}{ Glóbulos brancos $\left(\times 10^{3} \mathrm{~N} / \mathrm{mm}^{3}\right)$} \\
\hline TOTAL & $8,0 \pm 2,5$ & $8,0 \pm 2,7$ & $7,8 \pm 2,4$ & $7,9 \pm 2,8$ & $8,0 \pm 2,6$ \\
\hline CSA & $8,3 \pm 2,6$ & $8,1 \pm 2,7$ & $7,9 \pm 2,5$ & $7,8 \pm 2,9$ & $8,0 \pm 2,6$ \\
\hline $\mathrm{TAC}^{\mathrm{b}}$ & $7,2 \pm 2,1^{\mathrm{a}}$ & $7,5 \pm 2,3$ & $7,3 \pm 2,0$ & $8,7 \pm 2,1$ & - \\
\hline \multicolumn{6}{|l|}{ Plaquetas $\left(\times 10^{3} \mathrm{~N} / \mathrm{mm}^{3}\right)$} \\
\hline TOTAL $^{\mathrm{b}}$ & $208 \pm 74$ & $211 \pm 67$ & $220 \pm 63$ & $237 \pm 77$ & $237 \pm 75$ \\
\hline $\mathrm{CSA}^{\mathrm{b}}$ & $212 \pm 79$ & $217 \pm 70$ & $224 \pm 64$ & $238 \pm 78$ & $237 \pm 75$ \\
\hline TAC & $197 \pm 59$ & $191 \pm 52^{\mathrm{a}}$ & $198 \pm 50^{a}$ & $228 \pm 74$ & - \\
\hline
\end{tabular}

${ }^{a} \mathrm{p}<0,05$ ciclosporina (CSA) versus tacrolimo (TAC), comparando os valores laboratoriais para cada ano de estudo. ${ }^{\mathrm{b}} \mathrm{p}<0,05$, comparando os valores laboratoriais entre os anos após transplante renal em um mesmo grupo de tratamento. 
foram observadas variações significativas na contagem de glóbulos brancos. Valores menores que 3.000/ $\mathrm{mm}^{3}$ ocorreram em $1 \%$ dos receptores. Da mesma forma, apenas $1 \%$ a $2 \%$ dos pacientes apresentaram contagem de plaquetas inferior a $100.000 / \mathrm{mm}^{3}$.

\section{Dıscussão}

Os esquemas imunossupressores utilizando SRL e inibidores da calcineurina, sem terapia de indução, produziram sobrevidas do paciente $(92,8 \%)$ e do enxerto $(86,1 \%)$ ao final de 5 anos semelhantes àquelas observadas nos centros americanos, independentemente do esquema de imunossupressão utilizado (93\%, $80,9 \%) .{ }^{24}$ Considerando somente receptores tratados com SRL, as sobrevidas do paciente e do enxerto observadas no nosso estudo foram similares a outras séries publicadas quando o SRL foi combinado com CSA $(96,9 \%, 92,8 \%)^{25}$ ou TAC $(95,7 \% ; 90,8 \%) .{ }^{26} \mathrm{~A}$ incidência de rejeição aguda continua sendo um fator prognóstico importante para a sobrevida do paciente e do enxerto. ${ }^{27} \mathrm{~A}$ sobrevida cumulativa livre de rejeição aguda confirmada por biópsia foi de $82,2 \%$ após 5 anos, enquanto Krämer et al. observaram sobrevida livre de RACB, após 3 anos, de 78,8\% e 60,6\% em pacientes em uso de TAC e CSA, respectivamente. ${ }^{28}$ Esses dados comprovam a eficácia dessas estratégias imunossupressoras.

Tradicionalmente, as análises de eficácia e segurança de alternativas terapêuticas são realizadas por intenção de tratamento, não levando em consideração a frequência e a cronologia das mudanças de tratamento que ocorrem durante o período de observação. ${ }^{29}$ Quando avaliamos a eficácia de um regime imunossupressor após o transplante, o desfecho primário composto, definido como falha de tratamento, inclui tradicionalmente a incidência de rejeição aguda, perda do enxerto ou óbito do paciente. No nosso estudo, a incidência desse desfecho primário composto foi de $26,2 \%$ ao final do quinto ano de transplante. Entretanto, a incidência cumulativa de descontinuação do uso de SRL, uma variável adicional de eficácia, segurança e tolerabilidade do regime imunossupressor, foi de 33,2\% no mesmo período. Quando incluímos no desfecho primário composto a incidência de descontinuação do uso de SRL, a falha de tratamento foi de $47,8 \%$ ao final de 5 anos de transplante (Figura 1).

Apesar de não dispormos de grupo-controle comparativo ou de dados semelhantes publicados, algumas comparações ilustrativas podem ser feitas. Em um estudo recente que analisou padrões de mudança de medicação imunossupressora após transplante renal, dos pacientes recebendo inicialmente a combinação de CSA e SRL ou TAC e SRL, apenas 20,2\% e $18,5 \%$, respectivamente, ainda estavam recebendo essas combinações 4 anos após o transplante renal. ${ }^{30}$

A prevalência de interrupção do tratamento com SRL relativamente baixa sugere que sua tolerabilidade, utilizando-se esses regimes imunossupressores, foi elevada. As taxas de manutenção do tratamento, acima de $70 \%$ após o primeiro ano do transplante confirmam essa hipótese (Tabela 3). Além disso, a interrupção do uso da CSA foi substancialmente maior que do SRL, possivelmente com o objetivo de minimizar a nefrotoxicidade crônica. No total, 27,1\% dos pacientes necessitaram interrupção do uso do SRL em um intervalo de seguimento de 5 anos (Tabela 5). A incidência de interrupção do uso de SRL por reações adversas foi aproximadamente sete vezes maior do que a interrupção por falta de eficácia $(22,9 \%$ versus $3,3 \%$, Tabela 5). Essa incidência é menor do que a observada em outros estudos, nos quais a interrupção alcançou até $50 \% .{ }^{31}$ Entre as causas de interrupção, foram mais frequentes as reações adversas já conhecidas, como dislipidemia, disfunção do enxerto, proteinúria e dificuldade de cicatrização de feridas. A interrupção do uso de SRL foi semelhante entre os pacientes que receberam CSA ou TAC.

A interrupção definitiva do uso de um dos medicamentos imunossupressores está associada a um significante aumento na perda de enxerto. ${ }^{32}$ As taxas de interrupção do uso de SRL são variadas em diversas séries. Enquanto apresentamos taxa de interrupção de 27,1\% em 5 anos, nos EUA, 53,6\% dos receptores que iniciaram o esquema imunossupressor com CSA/ SRL e $56,7 \%$ daqueles que iniciaram com TAC/SRL continuaram com o mesmo esquema até 3 anos após o transplante. ${ }^{24}$ Estudo realizado em centro único mostrou uma taxa de interrupção do uso de SRL de apenas $13 \%$ após 10 anos de seguimento. ${ }^{7}$ Em comparação, fármacos contento ácido micofenólico apresentam taxas de interrupção de uso por intolerância que variam de $2 \%$ a $13 \%$ entre o primeiro e o terceiro anos após o transplante. ${ }^{32}$ Receptores cujo esquema imunossupressor inicial consiste em ácido micofenólico combinado a CSA ou TAC permaneceram com o mesmo tratamento em $63,5 \%$ e $70,9 \%$ dos casos, respectivamente, após 3 anos de transplante. ${ }^{24}$

Entre as possíveis razões para esses resultados, podemos destacar o tipo de transplante e o monitoramento clínico das concentrações dos fármacos imunossupressores. A maioria dos transplantes (92\%) foi 
realizada com rim de doador vivo, porém com órgãos de alta qualidade, em uma população de receptores relativamente jovem, com pouco tempo em diálise, baixa prevalência de portadores de diabetes mellitus e de comorbidades significativas. A redução precoce das doses e concentrações dos inibidores da calcineurina também pode ter sido decisiva. Ao final do primeiro ano, a média das concentrações de CSA $(77,9 \mathrm{ng} / \mathrm{mL})$ e de TAC $(6,2 \mathrm{ng} / \mathrm{mL})$ foram reduzidas, principalmente se consideramos o período em que tais transplantes foram realizados. Atualmente, dispomos de dados robustos que sugerem que mesmo concentrações ainda menores são eficazes e seguras. ${ }^{5,33}$ Além disso, a média da concentração de SRL foi em torno de $10 \mathrm{ng} / \mathrm{mL}$, abaixo daquela utilizada nos estudos iniciais com esse fármaco. Se, por um lado, o uso de doses reduzidas de inibidores da calcineurina pôde contribuir para a preservação da função renal, por outro lado, a manutenção de concentrações menores de SRL pôde aumentar a tolerabilidade e a segurança desses regimes imunossupressores.

A qualidade da função renal no final do primeiro ano do transplante está associada à sobrevida do enxerto. ${ }^{34}$ Neste estudo, a média da creatinina ao final do primeiro ano foi de $1,69 \mathrm{mg} / \mathrm{dL}$, não tendo sido observada deterioração significativa nos anos subsequentes, o que sugere um manuseio adequado desses regimes imunossupressores. As causas da diferença observada na função renal entre os pacientes que receberam CSA ou TAC são multifatoriais e difíceis de serem definidas com exatidão. A maior proporção de pacientes que interrompeu o uso da CSA ou que recebeu doses reduzidas de CSA pode ter contribuído para a diferença observada. Isso sugere que possa ser necessária também a interrupção do uso do TAC, bem como o seu emprego em doses mais reduzidas, para a preservação da função renal a longo prazo. Da mesma forma, a presença de proteinúria é um fator de risco associado à sobrevida do paciente e do enxerto em receptores de transplante renal. ${ }^{35} \mathrm{~A}$ média da concentração de proteína em amostra isolada de urina foi de $0,52 \mathrm{~g} / \mathrm{dL}$ no primeiro ano de transplante, com aumento gradual $(58 \%)$ até $0,82 \mathrm{~g} / \mathrm{dL}$ no quinto ano de transplante, sendo que nenhum paciente apresentou valores de proteinúria que ultrapassassem $1 \mathrm{~g} / \mathrm{L}$. Não podemos determinar se esse grau e incremento na proteinúria é consequência direta do uso de SRL, do desenvolvimento da disfunção crônica do enxerto, recorrência ou glomerulopatia “de novo" ou, o que é mais provável, uma combinação desses fatores.

Hipercolesterolemia e hipertrigliceridemia são fatores de risco associados a maior risco cardio- vascular tanto para a população geral quanto para os receptores de transplante renal, podendo, também, estar associados à nefropatia crônica do enxerto. ${ }^{36}$ Entretanto, a magnitude do aumento nas concentrações médias de colesterol e triglicérides foi pequena, apesar de maior entre os pacientes recebendo CSA, com tendência à redução após o primeiro ano do transplante. ${ }^{7,16}$

Finalmente, apesar da possibilidade de interferência do SRL sobre o metabolismo de carboidratos, a concentração média de glicose ficou dentro dos limites de normalidade, não se observando diferença entre os pacientes que receberam CSA ou TAC. Entretanto, a análise da incidência de diabetes mellitus após o transplante renal não foi um dos objetivos desse estudo.

Em resumo, a eficácia dos regimes imunossupressores utilizando SRL combinado a inibidores de calcineurina ficou estabelecida na população estudada. Apesar da segurança e da tolerabilidade terem sido adequadas, a maior causa para interrupção do uso de SRL foi a ocorrência de reações adversas. O aperfeiçoamento desses regimes deve visar, portanto, à redução ainda maior dessas reações adversas, seja com a introdução tardia do SRL após o transplante renal, para evitar as complicações perioperatórias, ou com a minimização ou eliminação do inibidor da calcineurina posteriormente, para preservar a função renal a longo prazo.

\section{REFERÊNCIAS}

1. Hartford CM, Ratain MJ. Rapamycin. Something Old, Something New, Sometimes Borrowed and Now Renewed. Clin Pharmacol Ther 2007; 82:381-8.

2. Ferreira AN, Machado PGP, Felipe CR et al. Concentrationcontrolled use of sirolimus (SRL) associated with reduced exposure of cyclosporine (CSA) in black recipients of primarily living renal allograft donors: 12-month results. Clin Transplant 2005; 19:607-15.

3. Felipe CR, Park SI, Machado PGP et al. Cyclosporine (CSA) and Sirolimus (SRL) pharmacokinetics and drugto-drug interactions in kidney transplant recipients. Fundam Clin Pharmacol. [in press] 2009.

4. Kahan BD. Efficacy of sirolimus compared with azathioprine for reduction of acute renal allograft rejection: a randomized multicenter study. The Lancet 2000; 356:194-202.

5. Kahan BD, Julian BA, Pescovitz MD, Vanrenterghem Y, Neylan J. Sirolimus reduces the incidence of acute rejection episodes despite lower cyclosporine doses in Caucasian recipients of mismatched primary renal allografts: a phase II trial. Transplantation 1999; 68:1526-32.

6. Podder H,StepkowskiSM, NapoliKL etal.Pharmacokinetic interactions augment toxicities of sirolimus / cyclosporine combinations. J Am Soc Nephrol 2001; 12:1059-71. 
7. Kahan BD, Knight R, Schoenberg L et al. Ten years of sirolimus therapy for human renal transplantation: The University of Texas at Houston experience. Transplant Proc. 2003; 35(supl.1):25s-34s.

8. Machado PGP, Felipe CR, Hanzawa NM et al. An openlabel randomized trial of the safety and efficacy of sirolimus vs. azathioprine in living related renal allograft recipients recipients receiving cyclosporine and prednisone combination. Clin Transplant 2004; 18:28-38.

9. Tedesco-Silva H, Medina-Pestana J, Alfieri F, Tai SS. A randomized open-label study to compare the safety and efficacy of cyclosporine (CSA) dose reduction with CSA elimination in de novo renal allograft patients receiving sirolimus. Transplantation 2008; 86(2S):157.

10. McAlister VC, Gao Z, Peltekian K, Domingues J, Mahalati K, MacDonald A. Sirolimus-tacrolimus combination immunosuppression. Lancet 2000; 355:376-7.

11 Cattaneo D, Merlini S, Pellegrino M et al. Therapeutic drug monitoring of sirolimus: effect of concomitant immunosupressive therapy and optimization of drug dosing. Am J Transplant 2004; 4:1345-51.

12. Park SI, Felipe CR, Pinheiro-Machado PG et al. Tacrolimus pharmacokinetic drug interactions: effect of prednisone, mycophenolic acid or sirolimus. Fundam Clin Pharmacol 2009; 23:137-45.

13. Sampaio EL, Pinheiro-Machado PG, Garcia R et al. Mycophenolate mofetil vs. Sirolimus in kidney transplant recipients receiving tacrolimus-based immunosuppressive regimen. Clin Transplant 2008; 22:141-9.

14. Webster AC, Lee VW, Chapman JR, Craig JC. Target of rapamycin inhibitors (sirolimus and everolimus) for primary immunosuppression of kidney transplant recipients: a systematic review and meta-analysis of randomized trials. Transplantation 2006; 81:1234-48.

15. Fonseca C, Felipe CR, Montegi SA et al. Conversion from cyclosporine/sirolimus to mycophenolate mofetil/ sirolimus combination in patients with chronic allograft dysfunction. Transplantation 2004; 78:260.

16. Grinyo JN, Alonso A, Arias M et al. Sirolimus use in de «novo renal» transplantation. Nefrologia 2006; 26(supl.2):33-51.

17. Straathof-Galema L, Wetzels JFM, Dijkman HBPM, Steenbergen EJ, Hilbrands LB. Sirolimus-associated heavy proteinuria in a renal transplant recipient: evidence for a tubular mechanism. Am J Transplant 2006; 6:429.

18. Izzedine H, Brocheriou I, Frances C. Post-transplantation proteinuria and sirolimus. N Engl J Med 2005; 353:2088

19. Keough-Ryan TM, Weerasinghe SD, Kirkland SA, Lawen JG, Kiberd BA. Comparison of sirolimus vs. cyclosporine based immunosuppressive therapy in renal transplant recipients. Am J Transplant 2003; 3:216.

20. Valente JF, Hricik D, Weigel K et al. Comparison of sirolimus vs. mycophenolate mofetil on surgical complications and wound healing in adult kidney transplantation. Am J Transplant 2003; 3:1128.

21. Kahan BD. Sirolimus-based immunosuppression: present state of the art. J Nephrol 2004; 17(Suppl.8):32-9.

22. Augustine JJ, Bodziak KA, Hricik DE. Use of sirolimus in solid organ transplantation. Drugs 2007; 67:369-91.
23. Di Marco GS, Andrade MCC, Felipe CR et al. Determination of sirolimus blood concentration using high-performance liquid chromatography with ultraviolet detection. Ther Drug Monit 2003; 25:558-64.

24. United States Department of Health and Human Services. Welcome to the 2007 OPTN / SRTR Annual Report: Transplant Data 1996-2006. Washington. [internet] [acesso em 01 de novembro de 2008]. Disponível em: http://www.optn.org/AR2007/

25. Gonwa TA, Hricik DE, Brinker K, Grinyo JM, Schena FP. Improved renal function in sirolimus-treated renal transplant patients after early cyclosporine elimination. Transplantation 2002; 74:1560-7.

26. Mendez R, Gonwa T, Yang HC, Weinstein S, Jensik S, Steinberg S. A Prospective, Randomized Trial of Tacrolimus in Combination with Sirolimus or Mycophenolate Mofetil in Kidney Transplantation: Results at 1 Year. Transplantation 2005; 80:303-9.

27. Harada KM, Sampaio ELM, Freitas TVS et al. Fatores de Risco Associados à Perda do Enxerto e Óbito Após o Transplante Renal. J Bras Nefrol 2008; 30:213-20.

28. Krämer BK, Del Castillo D, Margreiter R et al. Efficacy and safety of tacrolimus compared with ciclosporin A in renal transplantation: three-year observational results. Nephrol Dial Transplant 2008; 23:2386-92.

29. Fisher LD, Dixon DO, Herson J, Frankowski RK, Hearron MS, Peace KE. Intention to treat in clinical trials. In: Peace KE, editor. Statistical issues in drug research and development. New York: Marcel Dekker, Inc, 1990, pp. 331-50.

30. Meier-Kriesche, Chu AH, David HM, Chi-Burris K, Steffen BJ. Switching immunosuppression medications after renal transplantation - a common practice. Nephrol Dial Transplant 2006; 21:2256-62.

31. Oberbauer R, Segoloni G, Campistol JM et al. Early cyclosporine withdrawal from a sirolimus-based regimen results in better renal allograft survival and renal function at 48 months after transplantation. Transpl Intern 2005; 18:22-8.

32. Opelz G, Dohler B. Effect on kidney graft survival of reducing or discontinuing maintenance immunosuppression after the first year posttransplant. Transplantation 2008; 86:371-6.

33. Webster AC, Lee VW, Chapman JR, Craig JC. Target of rapamycin inhibitors (sirolimus and everolimus) for primary immunosuppression of kidney transplant recipients: a systematic review and meta-analysis of randomized trials. Transplantation 2006; 81:1234-48.

34. Hariharan S, Mcbride MA, Cherikh WS, Tolleris CB, Bresnahan BA, Johnson CP. Post-Transplant renal function in the first year predicts long-term kidney transplant survival. Kid Internat 2002; 6:311-8.

35. Amer H, Fidler ME, Myslak M et al. Proteinuria After Kidney Transplantation, Relationship to Allograft Histology and Survival. Am J Transplant 2007; 7:274856.

36. Akioka K, Takahara S, Ichikawa S, Yoshimura N, Akiyama T, Ohshima S. Factors Predicting Long-term Graft Survival after Kidney Transplantation: Multicenter Study in Japan. World J Surg 2005; 29:249-56. 